\title{
TRANSLATOR AND INTERPRETER TRAINING. CURRICULUM DESIGN. NEW PROSPECTS AND DILEMMAS
}

\author{
PIOTR KWIECINSKI, MARCIN FEDER \\ Adam Mickiewicz University - Poznan
}

\begin{abstract}
Institutional changes within universities and the challenge of EU enlargement present translator and interpreter trainers with both new opportunities and dilemmas. The major potential tension areas that must be addressed in the curricula include the status of retour translation and interpreting, the role of liaison interpreting in the curriculum, the separation of interpreter and translator training, and the focus on skills and techniques vs. background knowledge. The article suggests tentative solutions in these areas, based on the authors' own experience in curriculum design. The limitations and advantages of a postgraduate training programme within a foreignlanguage department are also discussed.
\end{abstract}

The aim of the present article is to outline the new prospects and dilemmas as well as tentative solutions in translator and interpreter (T/I) training curriculum design. While most of the issues discussed refer to the Polish context, they are likely to be valid for other Central European countries as well, since they are directly or indirectly related to Poland's current status as an EU applicant country. Beyond that, we hope that some of our observations and specific ideas on training and the curriculum will be of interest for T/I trainers in general. The chief source of data is our own recent experience of designing a thoroughly reformed curriculum of translator and interpreter training at a postgraduate (Master's) level in the School of English, Faculty of Modern Languages, Adam Mickiewicz University (IFA UAM) in Poznan, Poland.

\section{Translator and interpreter training within a foreign language department}

\subsection{The rationale}

There is little disagreement now that traditional degree programmes in foreign languages (such as MA or magisterium in English) offer very few, if any, of the skills needed for competent translation and interpreting. A growing awareness 
of that fact in the academic community, combined with the abrupt increase in demand for interpreters and translators on the Polish market in the wake of the political and economic changes in the early $1990 \mathrm{~s}^{1}$, have jointly contributed to establishing a number of postgraduate translation and interpreting schools. These schools are run by universities but are separated from language departments, and typically offer part-time training for a fee.

Without questioning the usefulness of these developments, our own institution adopted a somewhat different course. More specifically, it started offering a dedicated 4 to 6-semester training programme (initially in conference interpreting only) within the traditional five-year degree scheme, thus leading to a joint qualification of MA in English and a Diploma in Conference Interpreting. The reasons were mainly pragmatic and included: (a) the existing demand for high-quality interpreting and translation both from and into English (b) a growing pool of IFA staff who were themselves qualified and experienced interpreters and translators, developed an interest in T/I training, and increasingly felt that their skills and interests were underused within the traditional scheme, (c) the low course load during the final four semesters of the traditional five-year Master's programme, whereby contact hours are highly limited and students are mainly expected to work on their theses.

It was felt that the scheme could easily accommodate more intensive and more professionally oriented training, thus combining high academic standards with practical skills. Furthermore, we believed that offering a full-time foursemester programme in both (MA-level) English and interpreting represented a genuinely new quality ${ }^{2}$.

By definition, the $T / I$ training programme within our foreign-language department (IFA) had to fit into (or, perhaps, use creatively) the existing traditional institutional framework. Until 2003, the framework will continue to be based on a five-year MA in English degree scheme, out of which the three initial years are dedicated to common-core courses such as EFL, literature, history, institutions of English-speaking countries and linguistics, while the remaining two are devoted to seminar courses leading to an MA thesis in a selected subdiscipline (such as English literature or linguistics).

The T/I programme initially (1993-96) existed within that framework as an MA Seminar in Conference Interpreting. With the assistance of the TEMPUS grant, the programme was greatly expanded within the very same framework and

${ }^{1}$ See Kwieciński (2001), Chapter 2, for an extensive analysis of the implications of the Polish socio-economic transformation for translation.

${ }^{2}$ In Poland, another full-time degree programme in translation and interpreting is offered by the Institute of Applied Linguistics, Warsaw University (ILS UW). It involves a five-year MA programme in applied linguistics with later subdivisions into speciality areas including translation and interpreting. 
renamed specjalizacja magisterska (MA in English jointly with Translation and Conference Interpreting Programme) ${ }^{3}$.

The limitations and drawbacks of the above arrangement are fairly clear. So far, they have mainly included: (1) difficulties in formal recognition as a Т/I school due to our status as a department-internal "special option" scheme and the related restrictions on recruiting candidates outside IFA (till 2002, only students who had completed three years of common-core studies could qualify, subject to an internal admission procedure based on aptitude and competence tests); (2) the relative inflexibility and traditional nature of the language combination on offer, practically restricted to a specific AB (A. Polish B. English), supplemented by intensive training in other (specified) foreign languages ${ }^{4}$. The first of these limitations is likely to be eliminated in 2003 , as the final stage of the long-awaited institutional reform is implemented (see 1.2. below for details). The issue of the language combination has recently become less of a drawback, at least in the short run, due to the sustained demand of the local Polish market for a strong $\mathrm{AB}$ combination as well as the current needs of EU institutions (see esp. 2.1. below).

On the other hand, affiliation with a foreign-language department has proven to offer some benefits as well. First, the very nature of the English department and the unavoidable rigidity of the language combination encourage the focus on developing a strong B. Also, because candidates are all high-performing English students having completed 3 years of common-core EFL training, they are expected to have a near-native command of English (including authentic-sounding near-native pronunciation and usage). This proves to be an asset when a strong $B$ continues to be in demand both locally and internationally. Secondly, our status as (effectively) an MA programme allows full-time teaching, and consequently high training intensity and a greater course variety compared with part-time schemes (see 3.4. below).

\subsection{Imminent institutional changes and the curriculum}

Starting in 2003/04, in pursuance of the educational reform recently introduced in Poland, most university faculties, including ours, will offer a new two-tier model of university education, comprising three-year bachelor's programme (licencjat), op-

\footnotetext{
${ }^{3}$ Translator training was added as well a large variety of new courses. Next to simultaneous and consecutive interpreting, they now included content-organised $\mathrm{T} / \mathrm{I}$ courses in science and technology, business and law, as well as in terminology management and computer-assisted translation. Also, intensive training in (advanced) German and beginner Portuguese (as a "less widely spoken" language of the EU) started to be offered.

${ }^{4}$ Instruction in German-Polish translation and interpreting (German as C) was provided when the programme benefited from the TEMPUS scheme (1997-2002) and students were trained in the Institute of Translation and Interpreting of the University of Vienna for one semester. The $A B C$ option is now temporarily on hold because of institutional limitations and the current focus on a strong $\mathrm{AB}$ combination (see 2.1.).
} 
tionally followed by a two-year master's degree programme (magisterium), subject to a separate admission procedure. We aim to take advantage of the institutional reform to revamp our programme and re-launch it in 2003 in a new format complying with the two-tier system. The preparations will be preceded by a pilot project starting in October 2002. The major structural changes in our programme will include (a) since 2002, an introduction of two separate tracks, i.e. Conference Interpreting Programme (MACI) and the Translation Programme (MAT) ${ }^{5}$, sharing some common-core courses with the first semester additionally serving diagnostic and orientation purposes; (b) since 2003, opening the programme to any successful candidates holding the first university degree. The latter will radically change the status of the programme from a department-internal specialisation into a discrete postgraduate scheme.

\section{Tension areas and dilemmas in designing a future-oriented curriculum in Poland}

The reformed curriculum was inspired partly by our personal experience of the Monterey and Vienna schools and of the Interpreting Directorate of the European Parliament on the one hand, and our own practice on the Polish market on the other. It also sought to accommodate our new institutional status as a two-year postgraduate course. A combination of various goals and perspectives has created a number of areas which were either problematic or called for innovative solutions. These areas are discussed below.

\subsection{The status of retour interpreting}

Bi-active interpreting and translating is subject to much controversy both in Translation Studies and in the training practice, and the nativist model currently dominates in EU institutions (with the exception of Finnish). However, in a scenario outlined above, abandoning retour (translating and interpreting into an active foreign language) seems undesirable and unrealistic for a number of reasons. Firstly, translators and interpreters are routinely expected to work in both directions on the Polish market, where the demand is still almost exclusively for the $\mathrm{AB}$ type of competence (as opposed to ACC or ACCC). Secondly, due to the tradition of training and market realities, most teachers themselves have $\mathrm{AB}$ competence (or rarely $\mathrm{ABC}$ ). Last but not least, the imminent admission of a large group of CEECs

${ }^{5}$ As before, both programmes will lead to certificates in translation or conference interpreting respectively, and MA in English (which is due to nationwide legislative restrictions and university by-laws regarding degree diplomas). 
to the EU means that the current matrix system using the nativist model will no longer be sustainable in the EU institutions. Instead, either a radial system (bi-active interpreting combined with reliance on "pilot languages") or a hybrid system is most likely to be used in the near future (cf. e.g. Gomes 1999) ${ }^{6}$. Consequently translators/interpreters from CEECs with very strong B-language skills rather than an array of Cs will be in most demand. Hence, it seems that while an effort should be made to add Cs to the students' combinations, teaching of bi-active skills should be refined and extended, or even made a priority, particularly in the short term. This assumption is borne out, at least with respect to interpreting, in personal communication with representatives of EU interpreting services (The Interpretation Directorate of the European Parliament and the Joint Interpreting and Conference Service of the European Commission) on EU recruitment needs for the near future. It is also confirmed by the current EU freelance interpreter accreditation procedures, whereby strong Bs are currently given a clear preference over Cs.

\subsection{The status of liaison (dialogue) interpreting}

The status of liaison interpreting may soon begin to be changing due to a recent wave of interest in this area in Western European Translation Studies (cf. esp. Mason 1999) which may gradually percolate to training institutions. Polish training centres, including ours, have recently invested much effort into training first the trainers and then students in Western European standards of "proper" consecutive interpreting (including relevant note-taking skills), which was seldom taught in Poland until mid-1990s and is still rarely practised locally. While this effort is understandable and desirable, it has also meant relegating dialogue interpreting - once the most prominent next to simultaneous interpreting - to a peripheral role as a vestige of the past, even though it occupies an even larger space on the Polish market than in EU countries, since it continues to be used not only in courtrooms but also in many business settings. A tacit assumption is made that if students are taught proper consecutive interpreting, they will cope with liaison interpreting as its "simpler" variety, a claim which is yet to be verified. The challenge for curriculum design lies in reconciling all the conflicting and changeable factors involved.

${ }^{6}$ The "matrix" system of interpretation, which is currently used in EU institutions with the exception of Finnish, means interpreting exclusively from the floor (with no relay) and only into the interpreter's mother tongue. While it is widely held within the EU to be the only system to guarantee high interpretation quality, it requires all the official languages to be represented (as Cs) in each of the language booths. Under a hybrid system, the "old" booths would continue to operate as before, while booths representing languages of the new CEEC members would be bi-active, i.e. would provide interpretation into their respective mother tongues (Polish, Czech, etc.) for the floor, and into one of the pilot languages (most likely to include English) for relay. 


\subsection{The separation of translator and interpreter training vis-a-vis the Polish realities}

Until 2002, our curriculum was somewhat eclectic, with partly blurred distinctions between interpreter and translator training, in part reflecting the encompassing nature of the Polish concept of ttumacz (translator/interpreter). Students were trained in conference interpreting, but translation has also been used in some modules as a supplementary technique so as to offer them a "glimpse" of the profession. Experience shows that this is not entirely productive and future-oriented. A much clearer separation of the two skills in the new curriculum will allow both translator and interpreter trainees to maintain a clearer focus on their main field of study. Furthermore, it will allow us to invest more time in developing students' competence in a number of additional translation-specific skills, such as computer-assisted translation (CAT) and software localisation. This reflects anticipated Polish market realities in the area of translation, and especially localisation, where most of localisation into and out of Polish will have to be done in Poland given the status of Polish as a minority language (cf. e.g. Somers 1997 and Kwieciński 1998). Moreover, a number of Polish localisation companies have been already established. While recruiting in-house and freelance translators, they more and more frequently require at least basic tool literacy and the knowledge of underlying techniques. This separation of translator training is thus aimed at enhancing our graduates' chances on the very competitive and tight market (for a more detailed discussion on CAT training in the Polish setting see Feder 2002:6).

The need for the separation of the skills seems obvious enough. Yet we claim that complete separation would be counterproductive in specific areas. Legal translation and interpreting is a case in point. The area requires a special approach in the Polish context is due to the fact that Polish courts do not distinguish between interpreting court proceedings and translating legal documents, and require certified language experts to perform both tasks. The certification procedure now involves an examination in translating legal documents and in liaison interpreting, administered by a local court with seasoned court translators and interpreters serving on the examination board ${ }^{7}$. While no formal qualifications in interpreting and translation are currently required of the candidates, this is likely to change in the foreseeable future in view of the plans to introduce legislation establishing the so-called "public translator and interpreter" (cf. Ustawa z dnia... o ttumaczach publicznych. Projekt Ministra Sprawiedliwości. 1999 \& Wstepny Projekt RN PT TEPIS z dnia 29 listopada 1999 r. Ustawa o ttumaczach publicznych 1999). Public translators/interpreters will also be required to perform both tasks. A rigid separation of the two relevant skills in the curriculum appears to be incompatible with those requirements. Apart from the specific case of legal translation and interpreting, it seems

${ }^{7}$ This is true in most cases, since some courts unfortunately do not have any examination procedure in place. 
that retaining a common-core of courses that are taught jointly is desirable for a number of more general reasons as well. First, they may have an important orientation value in the initial stages of the two programmes, whereby students are exposed to some aspects of the other profession (i.e. translation students are required to perform some liaison interpreting and sight translation, whereas interpreting students are expected to translate some simple legal and administrative documents). Given skilful timetabling, students with no clear initial preference for either translation or interpreting, may even switch programmes during the initial period, based on their own preference and/or the teachers' advice. The second reason has more to do with plain logistics. Some courses can and should be taught jointly, thus freeing up teaching resources. Next to Legal Translation and Interpreting, such courses include: European and International Institutions, studies in the culture and institutions of relevant countries and language areas, Terminology Management, and Media Analysis (see 4 below for more curriculum details).

\subsection{Skills vs. content}

We subscribe to the view that meaningful interpreting and translating requires at least rudiments of systematic background knowledge, subsequently supplemented by intensive individual task-specific research in specialised areas. As suggested above, a full-time intensive four-semester postgraduate course can devote substantially more time to background studies than part-time courses, which often teach almost exclusively translation and interpretation techniques. Nonetheless, even an intensive full-time course must necessarily give priority to developing techniques at the expense of distinct systematic courses in specialist fields, such as law, economics or aspects of science and technology. It seems therefore that a flexible compromise solution should be sought.

\section{Tentative solutions}

When designing the new curriculum, we attempted to provide flexible compromise solutions in the areas identified above.

\subsection{Bi-active interpreting and the language combination}

We intend to continue placing much emphasis on retour skills for one language most likely to be on of the EU "pilot languages" in the future (English in our case). This appears to be an optimal solution in the short run. On the other hand, it seems that the programme should ideally extend beyond the age-old Polish 
tradition of $\mathrm{AB}$ training only and additionally offer a $\mathrm{C}$ language (or preferably a choice thereof), with the $\mathrm{ABC}$ model appearing to be the optimal compromise between Polish and European models. Implementing this model within the current institutional framework would require co-operation with other foreignlanguage departments which can provide qualified and experienced training staff. As a result a flexible joint $\mathrm{ABC}$ training programme could be developed, offering a range of combinations (e.g. A. Polish - B. English - C. German or A. Polish - B. German - C. English for a hypothetical case of a co-operative venture between just two departments).

\subsection{Dialogue interpreting in the curriculum}

Recognising that liaison (dialogue) interpreting is a complex skill requiring competence that is largely different from that required in conference interpreting (cf. esp. Mason 1999), we re-introduced it as one of the techniques into common-core modules, i.e. Legal Translation and Interpreting and Media Analysis. The former case has been discussed in some detail above. Media Analysis is a first-semester introductory course which is designed chiefly to sensitise students to current political, social and economic events both locally and worldwide, and to the need to keep abreast of such events on a regular basis in further training stages. Due to its nature, the course cannot use interpreting techniques that would require advanced notetaking and well-trained memory. Hence, it seems ideal for teaching the skills required in liaison (dialogue) interpreting, i.e. the variety of interpreting which often involves spontaneous, dialogic, face-to-face interaction (see e.g. Wadensjö 1998:81ff and passim) and where the interpersonal dimension (tenor) therefore plays a prominent role (see e.g. Tebble 1999:179-200 and Hale 1997:39-54). This mode of interpreting may be practised e.g. through student-prepared role-play based on current media themes.

\subsection{Programme separateness and overlap}

We have retained the eclectic approach for the three-semester legal course, which will be a required module for both tracks, in view of the realities of Polish certified translation and interpreting outlined above. As mentioned before, a range of other courses have been designed as part of the common-core course load.

\subsection{Background knowledge vs. professional skills}

(a) Interpreter training: We have retained and refined a number of contentoriented modules (Interpreting LSP Texts), parallel to traditional skill-oriented modules (Simultaneous Interpreting and Consecutive Interpreting) in the con- 
ference-interpreting programme. Content-oriented modules combine a number of interpreting techniques within one class (fluency practice, monolingual text reconstruction, liaison interpreting, consecutive interpreting without notes, consecutive interpreting with notes, sight translation, simultaneous interpreting), adjusted to students' current competence at a given stage. They focus on selected fields of science and technology (e.g. environmental technology or telecommunications). One of the aims of content-oriented modules is to teach the rudiments of relevant fields through interpreted presentations delivered by invited specialists, students or the trainer. Thus, the source texts are made to serve a dual purpose. The corollary of that is a compromise between professional realism and pedagogical progression (Kelly 1998), whereby the texts must gradually progress from semi-artificial general-reference materials that are pressed into service as texts to be interpreted (and a suitable communicative context is devised by the trainer) to authentic papers delivered at scientific conferences (whereby the authentic context is simulated in class).

Due to the demands placed on teaching resources, the fields of politics/social policy and business/economics are not separated into an additional content-oriented module in the MACI programme, as they traditionally constitute the staple content of all courses in Consecutive and Simultaneous Interpreting. However, such additional separation is also desirable in principle and could be an option as the programme grows.

(b) Translator training: In contrast to interpreter training, content focus seems to have traditionally predominated in translator training. Also, training has often focused on fields characterised by a substantial degree of transcultural conceptual identity ${ }^{8}$, such as economics or technology, while a large part of translation expertise is acquired and enhanced by compiling and mastering unequivocal terminological correspondences (such as pl. pszenżyto - en. triticale) and learning the underlying concepts. Moreover, many of the genres represented within the fields that are studied most intensively also display substantial structural congruity across the cultures involved (i.e. the text conventions are similar).

We accept the shift of focus as inherent in the nature of the two professions. Consequently, we have established two broad content-oriented modules within the MAT programme: science and technology and business/finance/economics. As subject-related research and the actual translating are done

${ }^{8}$ Clearly, any claims to "transcultural conceptual identity" may only be approached in relative terms and as a convenient shorthand term. Without engaging in the complexities of the philosophical debate on whether there is such a thing as an underlying translinguistic concept, we adopt a commonsensical trainer perspective. Viewed from such a perspective, there is clearly a much larger area of conceptual similarity or identity translating texts on, say, offset printing or extrusion than in texts on educational systems or regional cuisine. 
discussing relevant aspects of background knowledge in the field under study (e.g. wastewater treatment or the company balance sheet), which is a productive compromise solution that we sought.

On the other hand, we felt that exclusive dedication to the mode of translating described above from the earliest training stages may seriously distort the students' implicit theory of translating. To compensate for the partly inevitable content-focus in translation training, we designed a skill-focused translation course in the first semester in parallel to the content-oriented courses. The course is called Translation Strategies, and is dedicated to presenting typical difficulties and problem areas that are encountered by translators in a broad variety of texts and that are mostly due to the existence of disparate conceptual systems (such as culture-specific items) or to other textual phenomena such as wordplay. The course is also intended to raise students' awareness of cross-cultural differences in textual conventions. Context- and cotext-sensitive functional solutions in these areas are discussed and practised. Thus, the Translation Strategies course is primarily designed to encourage a view of translation as a dynamic "toolbox" activity rather than a substitution skill, a view that we feel to be the cornerstone of competent translation but which would perhaps be more difficult to get across otherwise. Thus, the course may be viewed as a "theory-meetspractice" undertaking, or a hands-on introduction to the functionalist paradigm.

The dynamic view of translation is required later during the course in Legal Translation and Interpreting, which also typically requires bridging disparate legal systems through translation but imposes certain restrictions on the choice of translation strategies and procedures due to the nature of the subject field and the related registers (e.g. radical cultural substitution is discouraged in favour of more exotic procedures in a number of legal contexts) ${ }^{9}$.

\section{REFERENCES}

Alexieva, B. (1997): A Typology of Interpreter-Mediated Events. The Translator. Vol. 3. No 2. 153-174.

Feder, M. (2002): Localisation in Poland. An overview of the Polish localisation industry. TermNet News (2002) 74. 1-7.

Gomes, M. (1999): The Post-Enlargement Scenario. Lingua Franca. Le Bulletin du Service d'Interprétation du Parlement Européen. Vol. 2. No. 9. 2-3.

Hale, S. (1997): The Treatment of Register Variation in Court Interpreting. The Translator, Vol. 3. No. 1 (1997). 39-54.

Kelly, D. (1998): Criteria for the selection of texts as classroom material at different levels of a university translator training programme: combining professional realism with pedagogical progression. A paper delivered at the EST Congress, Granada, Spain, 23-26 September 1998.

\footnotetext{
${ }^{9}$ See Kwieciński (2001:157ff.) for an extensive taxonomy of translation procedures.
} 
Kwieciński, P. (1998): Translation Strategies in a Rapidly Transforming Culture. A Central European Perspective. In: L. Venuti (ed.): Translation and Minority (special issue of The Translator, Vol. 4., No 2.). 183-206.

Kwieciński, P. (2001): Disturbing Strangeness. Foreignisation and domestication in translation procedures in the context of cultural asymmetry. Torun. Edytor.

Mason, I (1999): Introduction. In: I. Mason (ed.): Dialogue Interpreting. (special issue of The Translator, Vol. 5. No 2.). 147-160.

Somers, H.L. (1997): Machine Translation and Minority Languages. Translating and the Computer 19. London: Aslib.

Tebble, H. (1999): The Tenor of Consultant Physicians. Implications for Medical Interpreting. In: I. Mason (ed.): Dialogue Interpreting. (special issue of The Translator, Vol. 5. No 2). 179200.

Wadensjö, C. (1998): Interpreting as Interaction. London \& New York: Longman. 